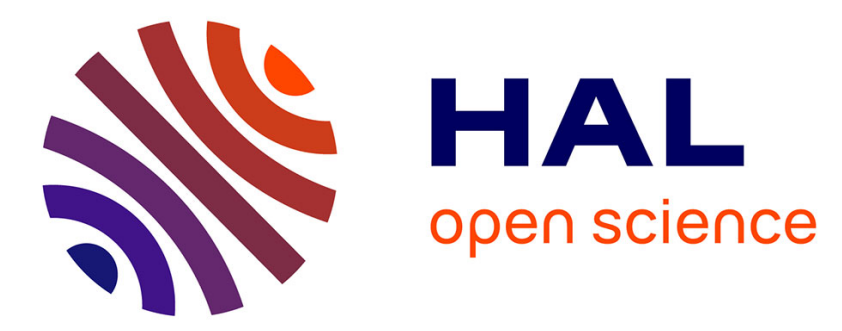

\title{
Étude de la répartition du champ magnétique dans la section droite d'une lentille magnétique multipolaire
}

\author{
Claude Audoin
}

\section{To cite this version:}

Claude Audoin. Étude de la répartition du champ magnétique dans la section droite d'une lentille magnétique multipolaire. Revue de Physique Appliquée, 1966, 1 (1), pp.2-10. 10.1051/rphysap:01966001010200 . jpa-00242680

\section{HAL Id: jpa-00242680 https://hal.science/jpa-00242680}

Submitted on 1 Jan 1966

HAL is a multi-disciplinary open access archive for the deposit and dissemination of scientific research documents, whether they are published or not. The documents may come from teaching and research institutions in France or abroad, or from public or private research centers.
L'archive ouverte pluridisciplinaire HAL, est destinée au dépôt et à la diffusion de documents scientifiques de niveau recherche, publiés ou non, émanant des établissements d'enseignement et de recherche français ou étrangers, des laboratoires publics ou privés. 


\title{
ÉTUDE DE LA RÉPARTITION DU CHAMP MAGNÉTIQUE
}

\section{DANS LA SECTION DROITE D'UNE LENTILLE MAGNÉTIQUE MULTIPOLAIRE}

\author{
Par Claude Audoin, \\ Institut d'Électronique, Faculté des Sciences, Orsay.
}

\begin{abstract}
Résumé. - Nous avons établi, dans l'hypothèse où les pôles sont équipotentiels, les expressions permettant le calcul de l'induction magnétique dans la zone utile de l'entrefer de lentilles magnétiques multipolaires, ainsi que du flux magnétique à travers les pôles.

Ces calculs s'appliquent au cas où les pièces polaires ont, en section droite, un profil constitué d'un arc de cercle tangent aux côtés d'un angle dont le sommet est sur l'axe de la lentille.

Des résultats numériques sont donnés pour des lentilles quadripolaires, hexapolaires, octopolaires.
\end{abstract}

Abstract. - Based on the hypothesis that the poles are équipotential, we have established the expressions leading to the determination of the magnetic-flux density in the useful region of the multipolar magnetic lens iron-gap, as well as the magnetic flux across the poles.

*These calculations concern the case where the poles show a cross section, whose profile consists of an arc of a circle tangential to the sides of an angle whose apex is on the axis of the lens.

Numerical results are given for quadripolar, hexapolar and octopolar lenses.

I. Introduction. - Des champs magnétiques à symétrie multipolaire sont couramment utilisés pour la focalisation de faisceaux d'ions ou d'électrons de hautes énergies, la séparation et la focalisation d'atomes ou de molécules neutres polarisés, et comme éléments correcteurs dans les grands accélérateurs circulaires de particules chargées.

Un champ magnétique à symétrie $2 N$-polaire $(N=2,3,4 \ldots$ pour une symétrie quadripolaire, hexapolaire, octopolaire ...) est produit par $2 N$ pièces polaires régulièrement réparties autour d'un axe de symétrie d'ordre $2 N$ et portées au potentiel $\pm V_{0}$, le signe étant alternativement positif ou négatif lorsqu'on passe d'une pièce polaire à la suivante par rotation autour de l'axe de symétrie.

Dans tout ce qui suit, nous désignerons par la lettre $V$, éventuellement indicée, le potentiel scalaire dont dérive, dans l'entrefer, l'induction magnétique $B$.

Dans l'entrefer l'équation de Laplace admet une solution qui, si les pièces polaires ont un plan méridien de symétrie dont l'un est pris pour origine angulaire, peut se mettre sous la forme $([1]$, p. 363, [2]) :

$\left\{\begin{array}{l}V / V_{0}=\sum_{p=0}^{\infty} A_{(2 p+1)}\left(r / r_{0}\right)^{(2 p+1) N} \cos (2 p+1) N \theta \\ r<r_{0}\end{array}\right.$

où les coordonnées $(r, \theta)$ sont les coordonnées polaires d'un plan de section droite, $r_{0}$ est le rayon du cercle de gorge de l'entrefer et $p$ un entier. Les coefficients $A_{\left(2_{p+1}\right)}$ dépendent de la forme des pièces polaires. Dans le cas particulier où elles ont un profil d'équation :

$$
\text { * } \quad\left(r / r_{0}\right)^{N} \cos N \theta=1
$$

le potentiel et l'amplitude de l'induction magnétique s'écrivent respectivement :

$$
\left\{\begin{array}{l}
V / V_{0}=\left(r / r_{0}\right)^{N} \cos N \theta \\
B / B_{0}=\left(r / r_{0}\right)^{N-1} \text { avec } B_{0}=N V_{0} / r_{0}
\end{array}\right.
$$

La symétrie est alors dite « $2 N$-polaire pure ».

Un profil défini par (2) est difficilement réalisable et son utilisation conduirait à des pièces polaires infiniment rapprochées loin de l'axe ; le flux magnétique serait alors court-circuité. Nous utilisons des pièces polaires faciles à réaliser limitées, en section droite, par deux droites faisant entre elles l'angle $2 \alpha$ et, vers l'extrémité effilée, par un arc de cercle tangent à ces deux droites (fig. 1) [3].

Cette géométrie limitée impose à $V$ de satisfaire à (1) et non plus à (3). Il est alors essentiel de connaître l'importance des harmoniques d'espace ainsi ajoutés à la répartition théorique cherchée. Ce problème n'est pas soluble directement de façon analytique pour le profil défini plus haut. Nous avons mis au point une méthode approchée de calcul qui nous permet, par l'étude d'un contour très voisin du profil pratique, et dans l'hypothèse où les pièces polaires sont équipotentielles, de déterminer rapidement l'écart de l'induction magnétique à la loi théorique (3), les coefficients des deux pre- 


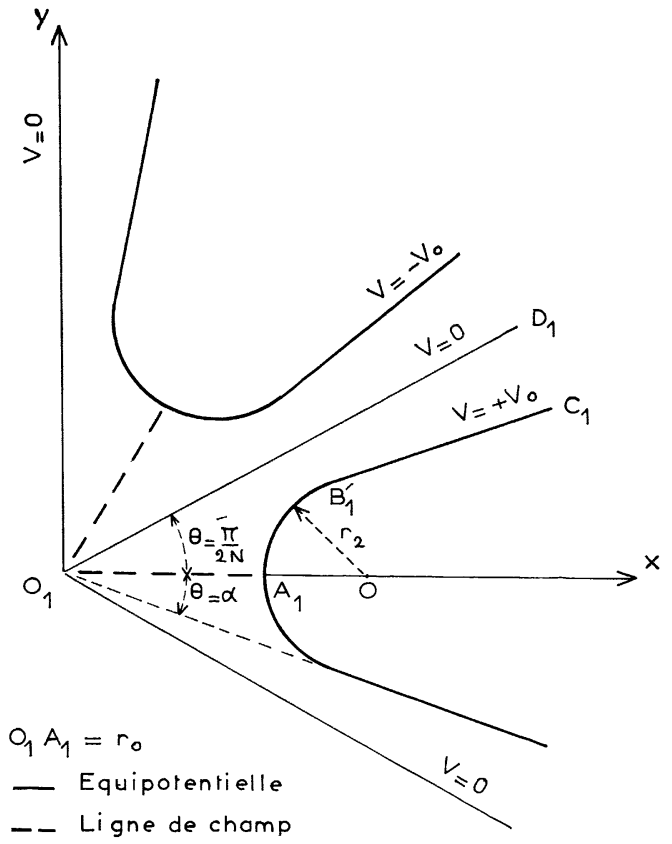

FIG. 1. - Profil des pièces polaires d'un aimant $2 N$-polaires.

miers termes du développement (1) et le flux magnétique à travers les pièces polaires.
2. Méthode de calcul. - Dans un plan de section droite, par raison de symétrie il suffit de considérer l'intérieur de l'angle $\left(\mathrm{O}_{1} \mathrm{x}, \mathrm{O}_{1} \mathrm{D}_{1}\right)$ (fig. 1). La région à étudier est limitée par :

- la demi-droite $\mathrm{O}_{1} \mathrm{D}_{1}$, correspondant au potentiel $V=0$;

- l'arc de cercle $\mathrm{A}_{1} \mathrm{~B}_{1}^{\prime}$ centré en $\mathrm{O}$ et la demi droite $\mathrm{B}_{1}^{\prime} \mathrm{C}_{1}$, au potentiel $V_{0}$;

- la ligne de champ $\mathrm{O}_{1} \mathrm{~A}_{1}$.

Un problème voisin a été résolu par $M$. Sonoda et al. [4] avec des pièces polaires limitées, à leur extrémité effilée, par un are de cercle centré en $\mathrm{O}_{1}$ ( $f$ g. 4 et 5). Comme ces Auteurs nous utilisons une suite de transformations conformes qui font correspondre la région étudiée à l'intérieur d'un condensateur plan.

Pour l'étude de la forme plus satisfaisante que nous avons adoptée nous remplaçons ( $f$ g. 2) l'arc de cercle $A_{1} B_{1}^{\prime}$ du contour réel par le contour analytique $A_{1} B_{1}$ (qui sera précisé par la suite, ainsi que son écart au contour réel). De plus, l'une des transformations conformes que nous utilisons est une transformation de Schwarz adaptée à la représentation d'un contour arrondi.

3. Transformations conformes utilisées. - 3.1 . Première transformation. - La transformation

$$
z=r_{0} \mathrm{e}^{w}
$$

fait correspondre le contour $\mathrm{O}_{2} \mathrm{~A}_{2} \mathrm{~B}_{2} \mathrm{C}_{2} \mathrm{D}_{2} \mathrm{O}_{2}$ du plan
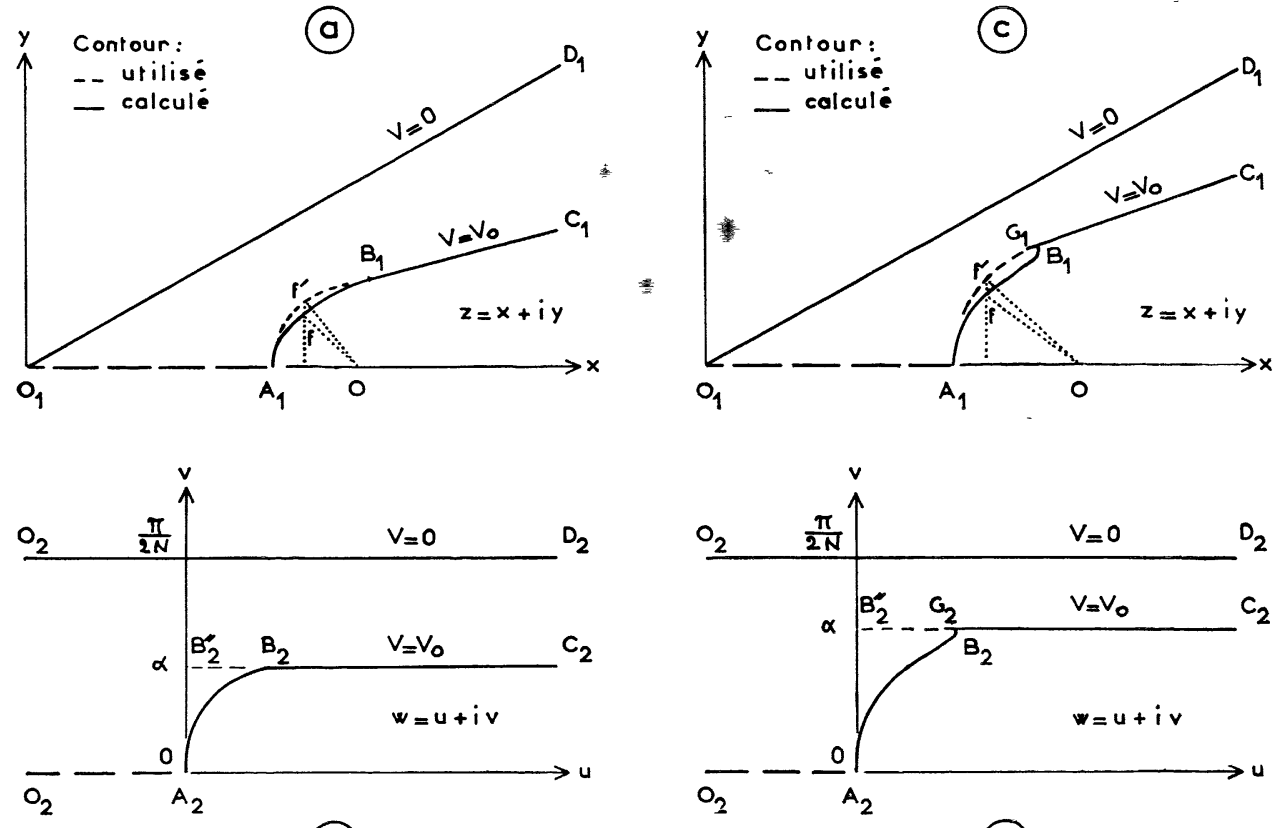

(b)

(d)

FIG. 2. - Correspondance entre le plan $z=x-i y$ et le plan $w=u+i v$ avec $z=r^{0} \mathrm{e}^{w}$ A gauche : $0<\alpha<\alpha_{0}$, à droite $: \alpha_{0}<\alpha<\pi / 2 N$. 
$w=u+i v(f i g .2)$ au contour $\mathrm{O}_{1} \mathrm{~A}_{1} \mathrm{~B}_{1} \mathrm{C}_{1} \mathrm{D}_{1} \mathrm{O}_{1} \mathrm{du}$ plan $z=x+i y$.

$\mathrm{O}_{2} \mathrm{~A}_{2}$ est une ligne de champ, $\mathrm{A}_{2} \mathrm{~B}_{2} \mathrm{C}_{2}$ est au potentiel $V_{0}, \mathrm{D}_{2} \mathrm{O}_{2}$ au potentiel zéro.

3.2. Deuxième transformation. - A) Cas $0<\alpha<\alpha_{0} .-1^{0}$ Établissons d'abord l'expression de la transformation de Schwarz qui fait correspondre à l'intérieur du polygone $\mathrm{O}_{2} \mathrm{~A}_{2} \mathrm{~B}_{2}^{\prime \prime} \mathrm{C}_{2} \mathrm{D}_{2} \mathrm{O}_{2}$ (fig. $2 b$ ) le demi plan $\eta>0$ (fig. $3 a$ ). Cette transformation s'écrit :

$$
\left\{\begin{array}{l}
\mathrm{d} s / \mathrm{d} t=K \Pi_{\mathrm{i}}\left(t-t_{\mathrm{i}}\right)^{\left(\frac{\alpha_{1}}{\pi}-1\right)} \\
t=\xi+i \eta
\end{array}\right.
$$

où $\alpha_{i}$ est l'angle intérieur du polygone, au sommet correspondant à $\eta=t_{\mathrm{i}}$.

Pour le polygone considéré, l'équation (5) devient :

$$
\mathrm{d} w / \mathrm{d} t=K \sqrt{t-a^{\prime}} /(t-1) \sqrt{t} .
$$

$2^{\circ}$ Selon une méthode proposée par H. W. Richmond ([5], [1], p. 352), si l'on remplace le terme $\sqrt{t-a^{\prime}}$ par $\sqrt{t-a}+\lambda \sqrt{t}$, lorsque $t$ est réel et varie entre 0 et $a$, la courbe $\mathrm{A}_{2} \mathrm{~B}_{2}$ est décrite dans le plan w. En effet, pour $0<n<a$ et $\xi=0$ :

$$
\operatorname{tg}\left[\operatorname{argt}\left(\frac{\mathrm{d} \psi}{\mathrm{d} t}\right)\right]=\operatorname{tg} \frac{\mathrm{d} v}{\mathrm{~d} u}=\operatorname{tg}\left(\frac{1}{\lambda} \sqrt{\frac{a-t}{t}}\right)
$$

varie progressivement de $+\infty$ à 0 quand on décrit le segment $\mathrm{A}_{3} \mathrm{~B}_{3}$ de l'axe réel du plan $t$.

La transformation qui fait passer du contour $\mathrm{O}_{2} \mathrm{~A}_{2} \mathrm{~B}_{2} \mathrm{C}_{2} \mathrm{D}_{2} \mathrm{O}_{2}$ au contour $\mathrm{O}_{3} \mathrm{~A}_{3} \mathrm{~B}_{3} \mathrm{C}_{3} \mathrm{D}_{3} \mathrm{O}_{3}$ est donc définie par :

$$
\frac{\mathrm{d} \omega}{\mathrm{d} t}=\frac{K}{t-1}\left(\lambda+\sqrt{\frac{t-a}{t}}\right) .
$$

$\mathrm{O}_{3} \mathrm{~A}_{3}$ est une ligne de champ, $\mathrm{A}_{3} \mathrm{C}_{3}$ est au potentiel $V_{0}, \mathrm{D}_{3} \mathrm{O}_{3}$ est au potentiel zéro.

$3^{\circ}$ Par intégration de (6) et compte tenu de la condition $w=0$ pour $t=0$, il vient avec $\log (-1)=i \pi$ :

$$
\begin{aligned}
\frac{w}{K} & =(\lambda+\sqrt{1-a}) \log (t-1) \\
& +\log \left[\frac{(\sqrt{t}+\sqrt{t-a})^{2}}{a}\right]
\end{aligned}
$$

$$
\begin{array}{r}
-\sqrt{1-a} \log \left[\frac{(\sqrt{t(1-a)}+\sqrt{t-a})^{2}}{a}\right] \\
-i \pi(1+\lambda) .
\end{array}
$$

$4^{\circ}$ Deux conditions déterminant les paramètres $K$, $a, \lambda$ sont $v=\alpha$ pour $a<t<1$ et $v=\pi / 2 N$ pour $t>1$. La troisième est liée à la forme de l'arrondi. Nous imposons à la courbe $A_{1} B_{1}$ d'avoir en $A_{1}$ le même rayon de courbure que l'arc de cercle $\Lambda_{1} B_{1}^{\prime}$ (c'est-à-dire $\left.r_{2}=r_{0} \sin \alpha /(1-\sin \alpha)\right)$. Le rayon de courbure $r_{2}$ de $\mathrm{A}_{1} \mathrm{~B}_{1}$ peut se calculer à l'aide de la relation $\frac{1}{r_{2}}=\frac{\partial}{\partial n}(\log |B|)$ avec $B$ donné par la formule (11) ou (11 bis). En $A_{1}$ il vient:

$$
r_{0} / r_{2}=-[1+(\lambda / 2 K a)] \text {. }
$$

Les relations déterminant les 3 paramètres sont done :

$$
\left\{\begin{array}{l}
\alpha=-K \pi(1-\sqrt{1-a}) \\
1 / N=-2 K(1+\lambda) \\
(1-\sin \alpha) / \sin \alpha=-[1+(\lambda / 2 K a)] .
\end{array}\right.
$$

Ce système admet une solution pour $0<\alpha<\alpha_{0}$, n'admet pas de solution pour $\alpha_{0}<\alpha<\pi / 2 N$. Le paramètre $a$ atteint la valeur 1 pour $\alpha=\alpha_{0}$. Les valeurs de $\alpha_{0}$ sont données dans le tableau 2 . Tous les calculs numériques sont effectués sur une machine arithmétique $\mathrm{CAB} 502 \mathrm{~B}$.

\section{TABLEAU 1}

$$
\frac{N}{\alpha_{0}} \quad \frac{2}{27^{\circ}} \frac{3}{1 / 10} \quad \frac{4}{18^{\circ}-2 / 10} \quad 13^{\circ} \frac{4}{7 / 10}
$$

B). Cas $\alpha_{0}<\alpha<\pi / 2 N$.

10 Pour passer du plan des $w$ au plan des $t$ nous adoptons alors la transformation définie par :

$$
\frac{\mathrm{d} s}{\mathrm{~d} t}=K\left[\left(1-\frac{1-a}{t-1}\right) \frac{1}{\sqrt{t} \cdot \sqrt{t-a}}+\frac{\lambda}{t-1}\right]
$$

(On retrouve la transformation (6) en changeant $1-a$ en $a-1$.) Cette nouvelle transformation fait correspondre à l'axe réel du plan $t$ le contour de la figure $2 d$. Lorsque $a<\eta<1$ le contour a un point de rebroussement correspondant à :

$$
\eta=\frac{2(2-a)-a \lambda^{2}-\sqrt{\left[2(2-a)-a \lambda^{2}\right]^{2}-4\left(1-\lambda^{2}\right)(2-a)^{2}}}{2\left(1-\lambda^{2}\right)}
$$

Dans le plan $z$ le contour obtenu est alors celui de la figure $2 c$. Nous verrons que $\mathrm{G}_{1} \mathrm{~B}_{1}$ est très petit pour les valeurs pratiquement intéressantes de $\alpha$. 
$2^{\circ}$ Par intégration de $(3$ bis $)$ et compte tenu de $\psi=0$ pour $t=0$, il vient :

$$
\begin{aligned}
\bar{K}= & (\lambda-\sqrt{1-a}) \log (t-1) \\
& +\log \left[\frac{(\sqrt{t}+\sqrt{t-a})^{2}}{a}\right] \\
& +\sqrt{1-a} \log \left[\frac{(\sqrt{t(1-a)}+\sqrt{t-a})^{2}}{a}\right] \\
& -i \pi(1+\lambda)
\end{aligned}
$$

$3^{0}$ Les conditions déterminant les paramètres $K$, $a, \lambda$ sont les mêmes que précédemment, c'eśt-à-dire : $v=\alpha$ pour $a<t<1, v=\pi / 2 N$ pour $t>1$, rayon de courbure $r_{2}$ en $A_{1}$ de la courbe $A_{1} B_{1}$ égal au rayon de l'arc de cercle $\mathrm{A}_{1} \mathrm{~B}_{1}^{\prime}$. Ici,

$$
r_{0} / r_{2}=-\left[1+\left(\lambda a / 2 K(2-a)^{2}\right)\right] .
$$

Les relations déterminant les 3 paramètres sont :

$$
\left\{\begin{array}{l}
\alpha=-K \pi(1+\dot{-1} \overline{1-a}) \\
1 / N=-2 K(1+\lambda) \\
(1-\sin \alpha) / \sin \alpha=-\left[1+\left(\lambda a / 2 K(2-a)^{2}\right)\right] .
\end{array}\right.
$$

Ce système admet en général deux solutions pour $\alpha_{0}<\alpha<\pi / 2 N$. Seule la solution pour laquelle le paramètre $a$ prend une valeur très proche de 1 conduit à un contour $\mathrm{A}_{1} \mathrm{~B}_{1}$ satisfaisant pour les

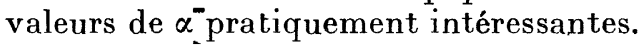
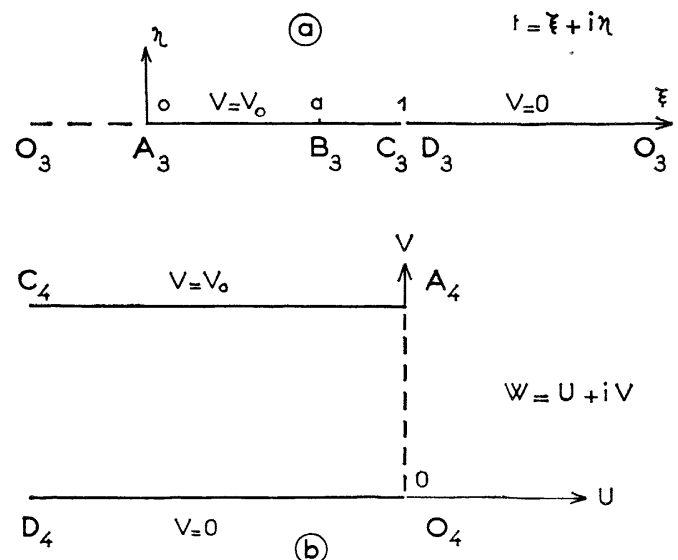

Fig. 3. - Contour transformé dans les plans $t$ et $W$.

3.3. Troisième transformation. - Nous passons du contour $\mathrm{O}_{3} \mathrm{~A}_{3} \mathrm{C}_{3} \mathrm{D}_{3} \mathrm{O}_{3}$ du plan $t$ ( f g g. $3 a$ ) au contour $\mathrm{D}_{4} \mathrm{O}_{4} \mathrm{~A}_{4} \mathrm{C}_{4}$ du plan $W=U+i V$ (fig. $\left.3 b\right)$ par la transformation de Schwarz définie par :

$$
\mathrm{d} W / \mathrm{d} t=C /[(t-1) \sqrt{t}]
$$

$\mathrm{D}_{4} \mathrm{O}_{4}$ est au potentiel zéro, $\mathrm{A}_{4} \mathrm{C}_{4}$ au potentiel $V_{o}$, $\mathrm{O}_{4} \mathrm{~A}_{4}$ est une ligne de champ et à l'intérieur du polygone la répartition de potentiel et les lignes de champ sont celles d'un condensateur plan. $V$ est la fonction potentiel ordinaire, $U$ la fonction flux.

Parintégration de (9), compte tenu de $W=0$ poür $t= \pm \infty$ et $W=i V_{0}$ pour $t=0$, il vient :

$$
W=\left(V_{0} / \pi\right) \log \sqrt{t}-1 / \sqrt{t}+1 .
$$

4. Écart entre le contour réel et le contour calculé - Les deux contours ne sont distincts que dans la partie arrondie. Nous appelons contour réel l'arc de cercle $\mathrm{A}_{1} \mathrm{~B}_{1}^{\prime}$ centré en $\mathrm{O}$.

$1^{\circ}$ L'arc de courbe $\mathrm{A}_{1} \mathrm{~B}_{1}$ (fig. $2 a$ ou $2 c$ ) a pour équation paramétrique :

$$
x=r_{0}{ }^{\mathrm{u}} \cos v \quad y=r_{0} \mathrm{e}^{\mathrm{a}} \sin v
$$

avec

$u=K \lambda \log \left(1-r_{1}\right)$

$v=-2 K\left[\operatorname{Arctg} \sqrt{\eta !\left(a-\gamma_{i}^{\prime}\right.}\right.$

$$
+\varepsilon \sqrt{1-a} \operatorname{Arctg} \sqrt{\eta(1-a) /(a-\eta)}]
$$

$0<\eta<a$

$\varepsilon=-1$ pour $0<\alpha<\alpha_{0}$

$\varepsilon=+1$ pour $\alpha_{f}<\alpha<\pi / 2 N$.

L'écart entre le contour réel et le contour calculé peut être caractérisé par la quantité :

$$
\Delta=1-\left(\mathrm{OF} / \mathrm{OF}^{\prime}\right)
$$

où $O$ est le centre de l'arc de cercle $A_{1} B_{1}^{\prime}, F$ appartient au contour calculé, $\mathrm{F}^{\prime}$ au contour réel. La valeur maximum de $\Delta$ est indiquée, pour différentes valeurs de $\alpha$, dans les tableaux $2 a, 2 b, 2 c$. Ces valeurs de $\alpha$ sont celles qui permettent de faire travailler dans des conditions satisfaisantes les

\section{TABLEAU $2 a$}

$$
\begin{aligned}
& \text { Symétrie Quadripolaire } \\
& \begin{array}{ccccccccc}
\alpha & 15^{\circ} & 1705 & 20^{\circ} & 22^{\circ} 5 & 25^{\circ} & 27^{\circ} 5 & 30^{\circ} & 32^{\circ} 5 \\
\underset{(\max }{(\text { en } \%)} & -0,9 & -0,4 & \overline{0,8} & \overline{1,9} & \overline{3,4} & \overline{6,1} & \overline{4,5} & -6,4
\end{array}
\end{aligned}
$$

TABLEAU $2 b$

SymÉtrie hEXAPOLAIRE

$\begin{array}{cccccc}\frac{\alpha}{\Delta \max } & 10^{\circ} & 12^{\circ} 5 & 15^{\circ} & 1705 & 20^{\circ} \\ (\text { en } \%) & -1 & 0,4 & 1,9 & 4,8 & 5,6\end{array}$

TABLEAU $2 c$

SyMÉtrie octopolaire

$\underset{\underset{(\text { en } \%)}{\alpha}}{\frac{7_{0}}{-1}} \quad \frac{10^{\circ}}{0,8} \quad \frac{12^{\circ} 5}{3,7} \quad \frac{15^{\circ}}{6}$


circuits magnétiques des lentilles multipolaires. Ceux-ci ont des performances optima pour $\alpha \# \pi / 4 N$.

Les figures 4,5 et 6 précisent la comparaison entre le contour calculé et le contour réel pour deux groupes de valeurs de $\alpha$ : d'une part $\alpha \# \pi / 4 N$ (deuxième transformation de type A) d'autre part $\alpha \# \alpha_{1}$ (deuxième transformation de type B). $\alpha_{1}$ est la valeur pour laquelle le rayon de l'arc de cercle $A_{1} B_{1}^{\prime}$ est égal au rayon de courbure en $A_{1}$ de l'équipotentielle théorique définie par l'équation (2). $\alpha_{1}$ est solution de l'équation sin $\alpha_{1}=1 / N$. Cette valeur est intéressante a priori car le contour réel est, sur une grande étendue angulaire, très voisin de l'équipotentielle théorique (2).

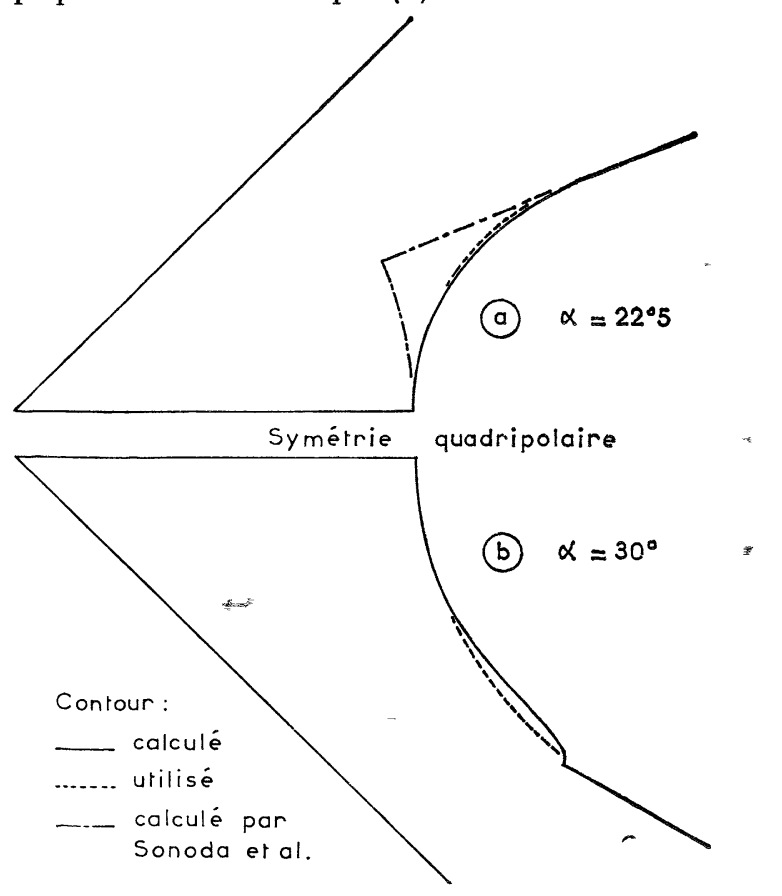

FIG. 4. - Symétrie quadripolaire.

Comparaison entre différents profils de pièce polaire.

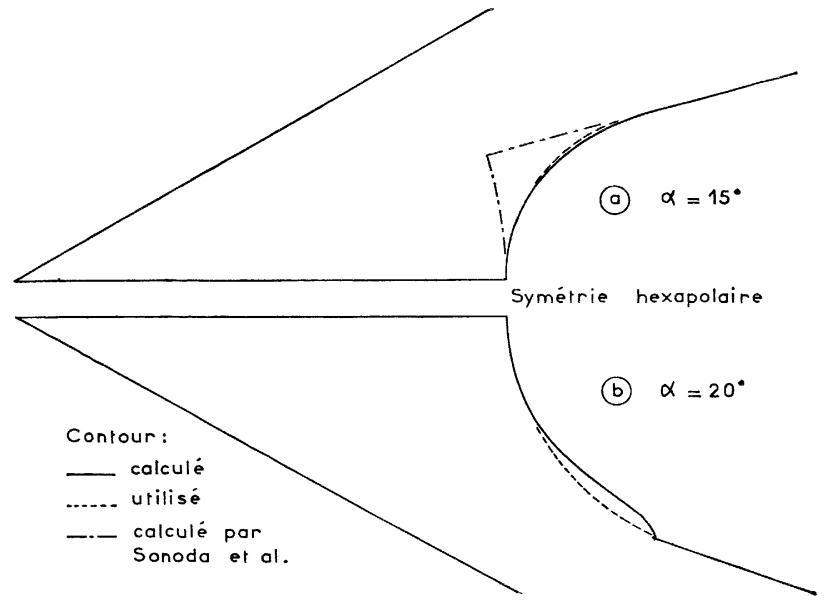

Fig. 5. - Symétrie hexapolaire.

Comparaison entre différents profils de pièce polaire.

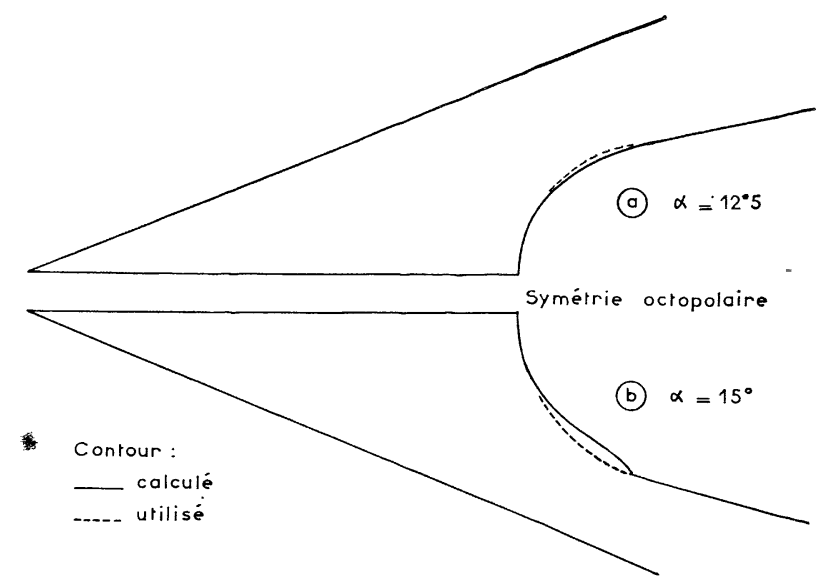

FIG. 6. - Symétrie octopolaire.

Comparaison entre différents profils de pièce polaire.

$2^{o}$ Dans le cas où $\alpha_{0}<\alpha<\pi / 2 N$ le contour calculé présente une corne $B_{1} G_{1}$, dont la longueur est indiquée dans le tableau 4.

\section{TABLEAU 3}

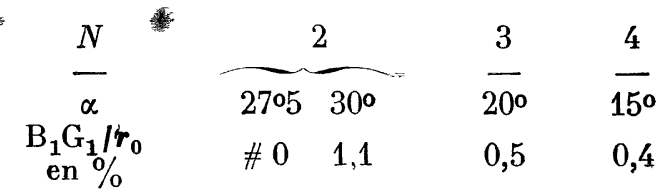

Pour $\alpha \# \alpha_{1}$ cette corne n'est pratiquement pas visible sur les figures 4,5 et 6 .

$3^{\circ}$ L'écart entre le contour réel et le contour calculé est donc petit pour les valeurs de $\alpha$ considérées.

5. Induction magnétique dans l'entrefer. - 5.1 . Expression de L'induction magnétique. - Dans le plan $z=x+i y$ de la figure 1 l'induction magnétique complexe est :

$$
B=B_{y}+i B_{x}=-\mathrm{d} W / \mathrm{d} z .
$$

A) $0<\alpha<\alpha_{0}$. Compte tenu des équations $(4),(6),(9)$ nous obtenons :

$$
\left\{\begin{array}{l}
B=\frac{B_{1} r_{0} \sqrt{a}}{z(\lambda \sqrt{t}+\sqrt{t-a})} \\
B_{1}=\frac{2 N / 1+\lambda) V_{0}}{\pi r_{0} \sqrt{a}} .
\end{array}\right.
$$

$B_{1}$ est l'induction magnétique en $A_{1}$, au sommet d'une pièce polaire.

B) $\alpha_{0}<\alpha<\pi / 2 N$. - Compte tenu des équations (4), (6 bis), (9) nous obtenons :

$$
\left\{\begin{array}{l}
B=\frac{B_{1} r_{0}(2-a) \sqrt{t-a}}{z \sqrt{a}(t-2+a)+\lambda \sqrt{t} \cdot \sqrt{t-a}} \\
B_{1}=\frac{2 N(1+\lambda) V_{0} \sqrt{a}}{\pi r_{0}(2-a)} .
\end{array}\right.
$$


Dans ces expressions de l'induction magnétique complexe, les variables complexes $t$ et $z$ sont reliés par les expressions (7), (7 bis), (4).

Dans les directions $\theta=0$ et $\theta=\pi / 2 N$, les parties réelles et imaginaires de (7) et $(7 \mathrm{bis})$ se séparent aisément. On obtient :

$-\operatorname{selon} \theta=0$

$\left\{\begin{array}{l}\eta<0, \xi=0 \\ r / r_{0}=\mathrm{e}^{u} \\ \frac{u}{K}=(\lambda-\varepsilon \sqrt{1-a}) \log (1-\eta) \\ +2 \log \left(\frac{\sqrt{-\eta}+\sqrt{a-\eta}}{\sqrt{a}}\right) \\ +2 \varepsilon \sqrt{1-a} \log \left(\frac{\sqrt{-\eta(1-a)}+\sqrt{a-\eta}}{\sqrt{a}}\right)\end{array}\right.$ $-\operatorname{selon} \theta=\pi / 2 N$

$$
\left\{\begin{array}{l}
\eta>1, \xi=0 \\
r / r_{0}=\mathrm{e}^{u} \\
\frac{u}{K}=(\lambda-\varepsilon \sqrt{1-a}) \log (\eta-1) \\
\quad+2 \log \left(\frac{\sqrt{\eta}+\sqrt{\eta-a}}{\sqrt{a}}\right) \\
\quad+2 \varepsilon \sqrt{1-a} \log \left(\frac{\sqrt{\eta(1-a})+\sqrt{\eta-a}}{\sqrt{a}}\right) .
\end{array}\right.
$$

Avec

\begin{tabular}{|c|c|c|c|c|c|c|c|c|}
\hline \multicolumn{9}{|c|}{ SYMÉtRIE QUADRIPOLAIRE } \\
\hline$\alpha$ & $15^{\circ}$ & 1705 & $20^{\circ}$ & 2205 & $25^{\circ}$ & 2705 & $30^{\circ}$ & 3205 \\
\hline$\overline{B_{1}} / B_{0}$ & $1 \overline{, 292}$ & $1, \overline{218}$ & $1, \overline{161}$ & $1, \overline{116}$ & $1, \overline{081}$ & $1, \overline{053}$ & $1, \overline{025}$ & $0, \overline{989}$ \\
\hline
\end{tabular}

$$
\begin{aligned}
& \varepsilon=-1 \text { pour } 0<\alpha<\alpha_{0} \\
& \varepsilon=+1 \text { pour } \alpha_{0}<\alpha<\pi / 2 N .
\end{aligned}
$$

\begin{tabular}{|c|c|c|c|c|c|}
\hline$\alpha$ & $10^{\circ}$ & $12^{\circ} 5$ & $15^{\circ}$ & 1705 & $20^{\circ}$ \\
\hline${ }_{1} / B_{0}$ & 1,290 & 1,184 & 1,112 & 1,061 & 1,019 \\
\hline
\end{tabular}

Les valeurs de $B_{1} / B_{0}$ sont portées dans les tableaux $4 a, 4 b, 4 c$.

\section{TABLEAU $4 a$}

TABLEAU $4 b$

Symétrie heXapolatre

5.2. Écart entre Les inductions magnétiQues CALCULÉE ET THÉORIQUe. - Nous caractérisons l'écart entre l'induction magnétique calculée à l'aide de la formule (11) ou (11 bis) et l'induction magné-

\begin{tabular}{|c|c|c|c|c|}
\hline$\alpha$ & 705 & $10^{\circ}$ & $12^{\circ} 5$ & $15^{\circ}$ \\
\hline$B_{1} / B_{0}$ & 1,289 & 1,156 & 1,074 & 1,017 \\
\hline
\end{tabular}

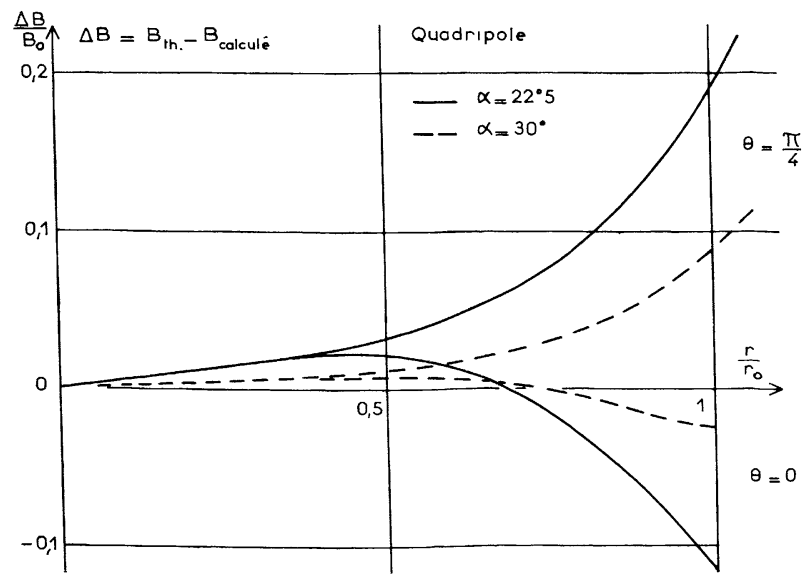

Fig. 7. - Symétrie quadripolaire. Écart entre les inductions magnétiques théorique et calculée, dans les directions $\theta=0$ et $\theta=\pi / 4$.
TABLEAU $4 c$

SYMÉtrie octopolaire

tique théorique obtenue lorsque l'équipotentielle $V_{\mathbf{0}}$ est une courbe d'équation (2) correspondant à la symétrie « $2 N$-polaire pure » par la quantité :

$$
\frac{\Delta B}{B_{0}}=\left(\frac{r}{r_{0}}\right)^{N-1}-\frac{|B(r, N)|}{B_{0}} .
$$

On passe de $B(t, N)$ donné par (11) ou (11 bis) à $B(r, N)$ en utilisant les expressions $(7)$ ou $(7 \mathrm{bis})$ et $(4)$.

Les figures 7, 8, 9 et 10 montrent cet écart calculé dans les directions $\theta=0$ et $\theta=\pi / 2 N$ pour différentes valeurs de $N$ et de $\alpha$.

Pour les valeurs de $\alpha$ considérées, les écarts diminuent lorsque l'angle $\alpha$ augmente.

6. Coefficients du développement du potentiel en termes multipolaires. - La détermination d'un grand nombre de coefficients du développement (1) nécessiterait le calcul du potentiel $V(\theta)$ pour $r=r_{0}$ et le développement de cette fonction en série de Fourier ([1], p. 364).

Nous nous sommes limités à la détermination plus simple, et suffisante en pratique, des coefficients 


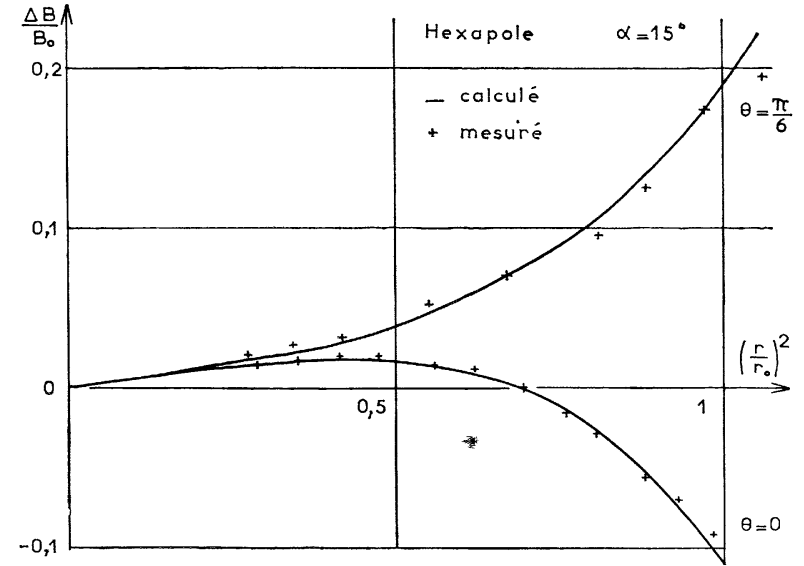

Frg. 8. - Symétrie hexapolaire. Écart entre les inductions magnétiques théorique et calculée d'une part, théorique et mesurée d'autre part pour $\alpha=15^{\circ}$.

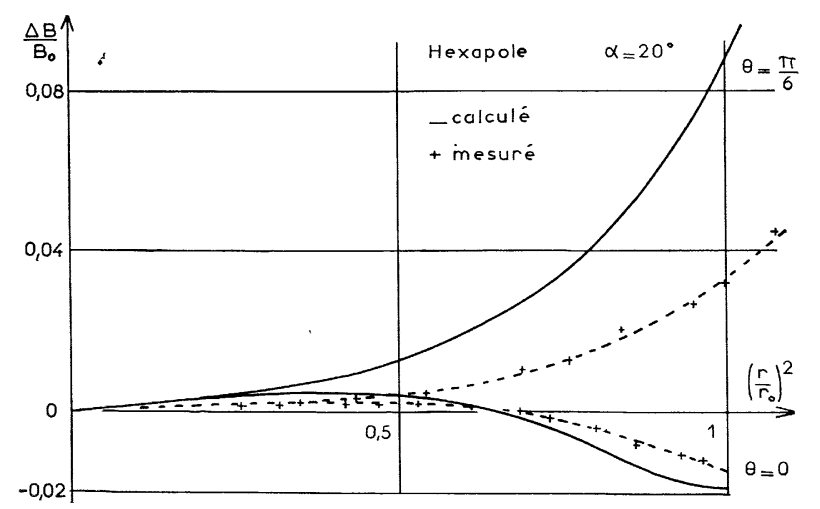

Fig. 9. - Symétrie hexapolaire. Écart entre les inductions magnétiques théorique et calculée d'une part théorique et mesurée d'autre part pour $\alpha=20^{\circ}$.

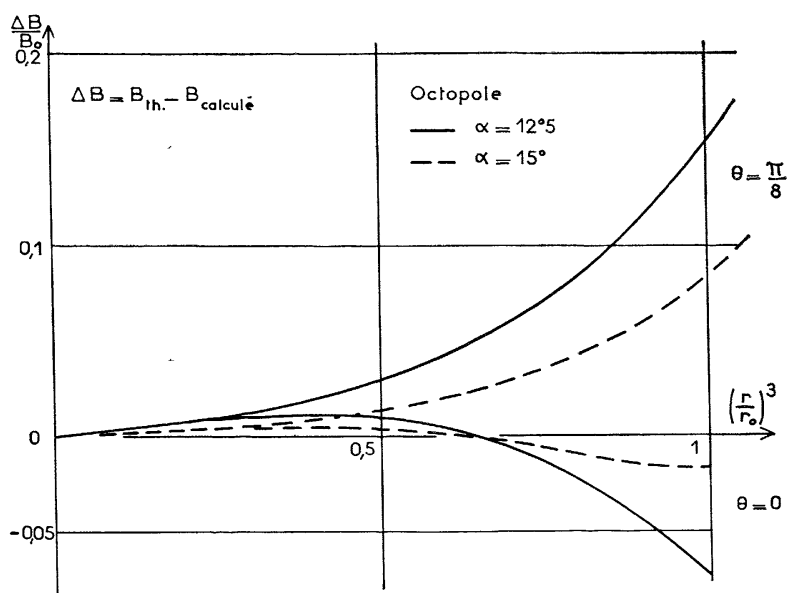

FIG. 10. - Symétrie octopolaire. Écart entre les inductions magnétiques théorique et calculée dans les directions $\theta=0$ et $\theta=\pi / 8$.

des deux premiers termes du développement à l'aide des résultats du paragraphe précédent.
Dans la direction $\theta=0$, l'induction magnétique est radiale, sa grandeur est, compte-tenu de (1)

$$
\begin{aligned}
\frac{|B|(\theta=0)}{B_{0}}=A_{1}\left(\frac{r}{r_{0}}\right)^{N-1} & +3 A_{3}\left(\frac{r}{r_{0}}\right)^{3 N-1} \\
& +5 A_{5}\left(\frac{r}{r_{0}}\right)^{5 N-1}+\ldots
\end{aligned}
$$

Le coefficient $A_{1}$ est calculé avec une bonne précision (10-4 environ) lorsque $r / r_{0}$ est suffisamment petit. Le coefficient $A_{3}$ est calculé pour une valeur de $r / r_{0}$ supérieure à la précédente, telle que d'une part, le deuxième terme de (12) ait alors un nombre suffisant de chiffres significatifs, et que d'autre part le troisième terme puisse être négligé. Ce coefficient est ainsi déterminé à quelques $10^{-2}$ près.

Les valeurs de $A_{1}, A_{3}$ sont rassemblées dans les tableaux $5 a, 5 b, 5 c$.

Pour toutes les valeurs de $\alpha$ considérées, dans la région centrale de l'entrefer, la grandeur de l'induction magnétique et son gradient sont inférieurs aux résultats théoriques déduits des formules (3).

TABLEAU $5 a$

Symétrie QUadripolaire

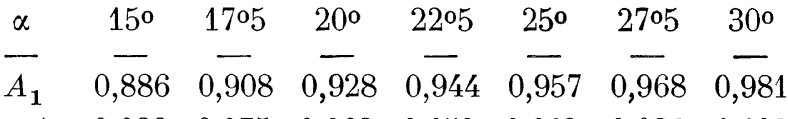

$$
\begin{aligned}
& \begin{array}{llllllll}
-A_{3} & 0,089 & 0,075 & 0,063 & 0,052 & 0,042 & 0,034 & 0,022
\end{array}
\end{aligned}
$$

\begin{tabular}{|c|c|c|c|c|c|}
\hline$\alpha$ & $10^{\circ}$ & 1205 & $15^{\circ}$ & 1705 & $20^{\circ}$ \\
\hline$A_{1}$ & 0,887 & 0,919 & 0,946 & 0,965 & 0,983 \\
\hline$-A_{3}$ & 0,087 & 0,067 & 0,047 & 0,035 & ,020 \\
\hline
\end{tabular}

TABLEAU $5 b$

SyMÉTRIE hEXAPOLAIRE

\begin{tabular}{|c|c|c|c|}
\hline$\alpha$ & 705 & $10^{\circ}$ & 1205 \\
\hline$\overline{A_{1}}$ & $0 \overline{8,886}$ & $\overline{0,929}$ & $\overline{0, \overline{9}} 0$ \\
\hline$-A_{\mathrm{s}}$ & 0,090 & 0,064 & 0,041 \\
\hline
\end{tabular}

TABLEAU $5 c$

SYMÉTRIE OCTOPOLAIRE

7. Flux à travers les pièces polaires. - La connaissance du flux magnétique à travers les pièces polaires est nécessaire à la détermination du circuit magnétique d'une lentille multipolaire.

La partie $B_{1} C_{1}$ du profil d'une pièce polaire est décrite lorsque $\xi=0$, quand $\eta$ varie entre $a$ et 1 . La fonction flux s'écrit alors :

$$
\frac{U}{V_{0}}=\frac{1}{\pi} \log \frac{1-\sqrt{\eta}}{1+\sqrt{\eta}}
$$


et $\eta$ est relié à $r / r_{0}$ par l'intermédiaire des formules (4),,$(7)$ ou (7 bis).

7.1. Expression approchée déduite des ForMULES ÉTABLIES. - Le paramètre $a$ étant voisin de $1\left(N=3, \alpha=15^{\circ}, a=0,982 ; N=3, \alpha=20^{\circ}\right.$, $a=0,994)$, l'expression (13) peut être écrite :

$$
U / V_{\mathbf{0}}=(1 / \pi)[\log (1-\eta)-\log 4]
$$

et nous posons $t=1$ dans la formule (7) ou (7 bis)

En tenant compte de plus de (8) ou ( 8 bis) nous obtenons :

$$
-\frac{U}{V_{0}}=C_{1}+\frac{1}{(\pi / 2 N)-\alpha} \log \frac{r}{r_{0}}
$$

avec

$$
\begin{aligned}
C_{1}=\frac{1}{\pi} \log 4 & \\
& -\frac{2 K}{(\pi / 2 N)-\alpha}\left[\log \left(\frac{1+\sqrt{1-a}}{\sqrt{a}}\right)\right. \\
& \left.+\varepsilon \sqrt{1-a} \log 2 \sqrt{\frac{1-a}{a}}\right] \\
\varepsilon= & -1 \text { pour } 0<\alpha<\alpha_{0} \\
\varepsilon= & +1 \text { pour } \alpha_{0}<\alpha<\pi / 2 N .
\end{aligned}
$$

Dans le cas d'une lentille hexapolaire l'expression approchée (14) est valable à $1 \%$ près pour $r / r_{0}=1,35$ si $\alpha=15^{\circ}$ et $r / r_{0}=1,45$ si $\alpha=20^{\circ}$; pour des valeurs supérieures de $r / r_{0}$ elle tend rapidement vers l'expression exacte.

7.2. Comparaison aVEc UN RÉSUltat obtenu par Une autre méthode. - Par ailleurs [3], pour la discussion des caractéristiques du circuit magnétique d'une lentille hexapolaire, nous avons fait une hypothèse simplificatrice sur la forme des lignes de champ dans l'entrefer qui conduit à l'expression simple :

$$
-\frac{U}{V_{0}}=C_{2}+\frac{1}{(\pi / 2 N)-\alpha} \log \frac{r}{r_{0}}
$$

avec

$$
C_{2}=\operatorname{tg} N \alpha+\frac{1}{(\pi / 2)-N \alpha} \log (\cos N \alpha) .
$$

Pour une lentille hexapolaire,

$$
\begin{array}{lll}
-\operatorname{si} \alpha=15^{\circ} & C_{1}=0,640 & C_{2}=0,559 \\
-\operatorname{si} \alpha=20^{\circ} & C_{1}=0,362 & C_{2}=0,408 .
\end{array}
$$

L'écart constant en valeur absolue entre les expressions (14) et (15) correspond à un écart relatif qui, pour $\alpha=15^{\circ}$, vaut $2 \%$ si $r / r_{0}=2,4$ et $1 \%$ si $r / r_{0}=7$ et qui, pour $\alpha=20^{\circ}$, vaut $2 \%$ pour $r / r_{0}=1,4$ et $1 \%$ si $r / r_{0}=2,1$.

La validité de l'expression (15) et des considérations qui en dérivent peut donc être 'étendue aux pièces polaires très courtes. Ce résultat est important car il permet de simplifier l'étude du circuit magnétique de lentilles multipolaires à pièces polaires très courtes et donc d'encombrement sensiblement moindre que les réalisations actuelles.

\section{Détermination expérimentale de l'écart entre} le champ réel et le champ théorique.

8.1. Méthode DE MEsure. - Nous avons déterminé à la cuve rhéographique la répartition de potentiel dans l'entrefer d'une lentille hexapolaire avec $\alpha=15^{\circ}$ et $\alpha=20^{\circ}$.

La répartition réelle étant voisine de la répartition théorique (3) nous avons adopté une méthode qui, grâce au théorème de superposition, fait directement apparaître Jeur écart [6].

Le modèle ( $f g .11$ ) représente un secteur limité par les demi-plans $\theta=+\pi / 6$ et $\theta=-\pi / 6$. Le contour MNP reproduit la forme réelle de l'extrémité d'une pièce polaire.

Considérons un premier état, correspondant à la situation réelle, où les électrodes $\mathrm{OQ}$ et $\mathrm{OL}$ sont au potentiel zéro, où les points du contour MNP sont au potentiel $V_{0}$, ceux situés sur LM et $\mathrm{PQ}$ à un potentiel que l'on peut évaluer approximativement comme le potentiel à l'intérieur d'un drièdre d'angle $(\pi / 6)-\alpha$ dont les côtés sont aux potentiels 0 et $V_{0}$. L'approximation ainsi faite n'influe pratiquement pas sur le résultat final si $L M$ et $P Q$ sont assez éloignés de la partie arrondie. Dans cet état le potentiel $V_{1}(r, \theta)$ est le potentiel cherché.

$\mathrm{Si}$, dans un second état, chaque point du contour OLMNPQO est porté à un potentiel calculé selon la relation (3), en tout point de la zone étudié le potentiel $V_{2}(r, \theta)$ est celui qui correspond à la symétrie hexapolaire pure. Dans cet état, le potentiel est nul sur OL et OQ, égal au $V_{0}$ au sommet du pôle.

Par superposition des deux états, on obtient le potentiel $V_{2}-V_{1}$, qui est l'écart à la loi théorique, rapporté au potentiel $V_{0}$. Il peut être mesuré à la cuve rhéographique si l'on impose aux frontières de la zone étudiée le potentiel correspondant à la superposition des conditions aux limites des deux états décrits.

Lorsque $\alpha=20^{\circ}$, l'écart à mesurer est très faible. Nous avons alors opéré en deux étapes : tout d'abord le potentiel des points situés sur $L^{\prime} M^{\prime}$ et $P^{\prime} Q^{\prime}$ a été mesuré dans les conditions indiquées ci-dessus, puis la zone à étudier a été réduite à la région limitée par le contour $\mathrm{OL}^{\prime} \mathrm{M}^{\prime} \mathrm{NP}^{\prime} \mathrm{Q}^{\prime} \mathrm{O}$.

L'allure générale des équipotentielles est représentée sur la figure 11 .

L'écart entre les inductions magnétiques réelle et théorique est déduit des cartes de potentiel.

8.2. Résultats. - La comparaison (fig. 8 et 9) entre les écarts mesurés et calculés fait apparaître un excellent accord pour $\alpha=15^{\circ}$. 


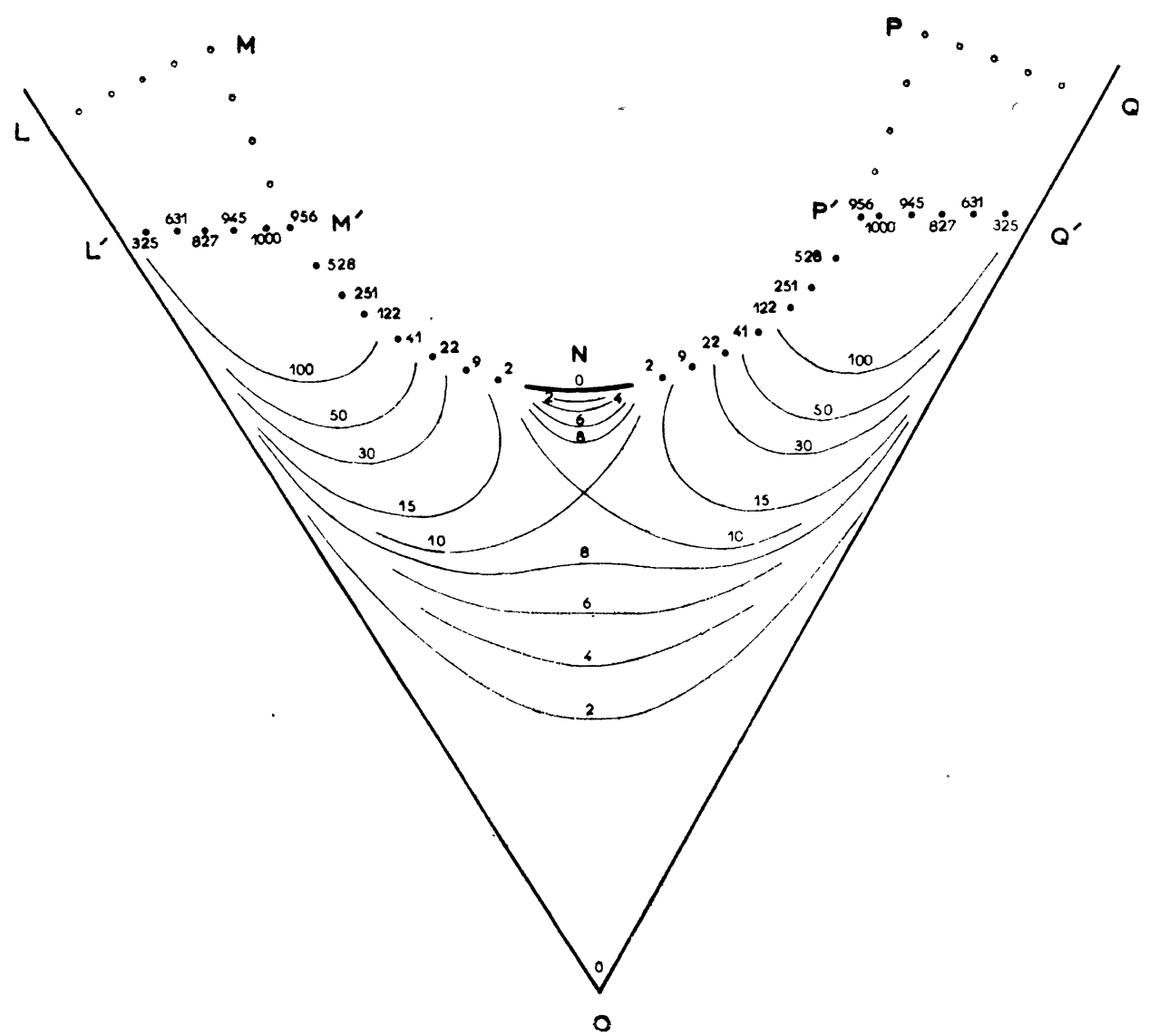

FIG. 11. - Carte de potentiel établie à la cuve rhéographique pour $\alpha=20^{\circ}$.

Écart à la rèpartition théorique correspondant à $V_{1}=V_{2}=3700$ au sommet du pôle.

Pour $\alpha=20^{\circ}$ ces écarts sont faibles. Le remplacement du contour réel par un contour analytique voisin d'une part, les incertitudes de mesure d'autre part (en particulier les différences de potentiel à mesurer à la cuve rhéographique ne sont pas grandes devant les d. d. p. de contact parasite entre électrode et électrolyte), introduisent sur ces écarts des erreurs relatives non négligeables. Une détermination plus précise nécessiterait l'utilisation d'une méthode numérique de résolution de l'équation de Laplace, avec un réseau à mailles serrées.

9. Conclusion. - Les résultats obtenus permettent d'avoir rapidement une vue d'ensemble sur la répartition de potentiel magnétique dans l'entrefer de lentilles magnétiques multipolaires dont les pièces polaires ont une forme géométrique simple, avec extrémité arrondie.

Ils autorisent en outre une discussion simple des propriétés du circuit magnétique d'aimants multipolaires à pièces polaires très courtes selon une méthode qui a déjà donné satisfaction lorsque ces pièces polaires sont longues.

L'évaluation quantitative de la correction à apporter éventuellement pour tenir compte de la perméabilité finie du matériau magnétique utilisé devra être envisagée dans chaque cas particulier, car elle dépend à la fois de la nature du matériau utilisé et de l'intensité maximum de l'induction qu'on veut obtenir en un point donné.

Manuscrit reçu le 18 octobre 1965.

\section{BIBLIOGRAPHIE}

[1] Durand (E.), Électrostatique et Magnétostatique, Masson et Cie, éditeurs, 1953.

[2] Schnuriger (J. C.), J. Physique Rad., 1961, 22, $68 \mathrm{~A}$.

[3] Audoin (C.), J. Physique, février 1965, 26, 71 A.
[4] Sonoda (M.), Katase (A.), Seki (M.) et Akiyoshi (T.), Nuclear Instruments and Methods, 1961, 12 349.

[5] Richmond (H. W.), Proc. London Math. Soc., 1923, Ser. 2, 22, 389.

[6] Rapport C. E. R. N., PS/MN 31, mars 1957. 